\title{
Charming bound states in a symmetry preserving contact interaction
}

\author{
Marco A. Bedolla ${ }^{a, *}$ \\ ${ }^{a}$ Facultad de Ciencias en Física y Matemáticas (FCFM), Universidad Autónoma de Chiapas, \\ Carretera Zapata Km. 8, Rancho San Francisco, Tuxtla Gutiérrez 29050, Chiapas, México \\ E-mail: marco.bedolla@unach.mx
}

\begin{abstract}
A collection of different studies about bound states of hadrons composed of charm quarks is presented. These studies used a symmetry preserving vector-vector contact interaction model within the framework of Schwinger-Dyson and Bethe-Salpeter equations. Despite its limitations, the contact interaction model offers a simple-to-implement alternative to perform exploratory studies of QCD, obtaining an spectrum of charmed hadrons in agreement within $2 \%$ when they are compared with experimental data. In particular, the spectrum of charm and beauty mesons is presented.
\end{abstract}

\footnotetext{
*** 10th International Workshop on Charm Physics (CHARM2020), ***

*** 31 May - 4 June, 2021 ***

*** Mexico City, Mexico - Online ***
}

\footnotetext{
${ }^{*}$ Speaker
} 


\section{Introduction}

The Schwinger-Dyson and Bethe-Salpeter equations framework has proven to be a reliable instrument to explain a wide range of QCD phenomena [1-3]. However, Maris et al. [4], or more recently Chen et al. [5], pointed out that brute force numerical evaluations depict problems when evaluating at large momentum transfer regions. And some of the Schwinger-Dyson community efforts consist in solving these problems from other perspectives [6-9].

In 2010, the contact interaction (CI) model appeared as an alternative to full QCD studies. It was first introduced by Gutierrez et al. [10-14] while studying masses, decay constants and form factor of light pseudoscalar and vector mesons. In this model, quarks interact not via mass-less vectorboson exchanges, but instead through a symmetry preserving vector-vector contact interaction. This interaction, along with the Schwinger-Dyson and Bethe-Salpeter equations in the rainbow-ladder approximation, implements confinement through a proper time regularization scheme [15]. A fully consistent treatment of the contact interaction model is simple to execute, and it produces useful results that can be compared and contrasted with full QCD calculations and experimental data.

The CI model was introduced as a simple model that could provide exploratory studies to QCD without the necessity of high performance computing [10]. The model was originally implemented to compute the spectrum, decay constant and electromagnetic form factor of pion, rho mesons and their diquark partners $[11,13]$. Successive works expanded this model to study unflavored heavy systems [16, 17], heavy-light systems [18, 19] and baryons [14, 20-22]. In these studies, there is a general consensus on obtaining that the mass spectrum of mesons and baryons is in a good agreement with experimental data. However, the vector decay constants are close to 50\% below the expected value [16].

Elastic and transition form factors have been computed for unflavored mesons, but the computations results on harder form factors as described in Refs. [13, 17, 23]. In addition, the CI model could be also implemented to study heavy tetraquarks via gluon exchange [24], instead of a quark exchange interaction in baryons [14], or light tetraquarks [25].

In this work, a summary of different studies employing the CI model to study charming and beauty bound states ${ }^{1}[16,17,22-24]$ is presented. When available, they will be compared to experimental data [26, 27]. For a complete model revision, and a comparison with fully-covariant models setups [28-31], it is recommended to check to Ref. [22].

\section{The Contact Interaction Model}

In this work, the Schwinger-Dyson and Bethe-Salpeter equations are used to solve the boundstate problem in terms of their building blocks (quarks), and their interactions with gluons. In order to solve a meson bound state equation, it is needed to know the quark propagator, the gluon propagator and the quark-gluon interaction. In the CI model, it is assumed that the quark-gluon interaction is led by symmetry-preserving vector-vector contact interaction; here, quarks are attached

${ }^{1}$ Composed of charm and bottom quarks 
through an interaction defined as:

$$
\begin{aligned}
g^{2} D_{\mu \nu}(k) & =\frac{4 \pi \alpha_{\mathrm{IR}}}{m_{g}^{2}} \delta_{\mu \nu} \equiv \frac{1}{m_{G}^{2}} \delta_{\mu \nu}, \\
\Gamma_{\mu}^{a}(p, q) & =\frac{\lambda^{a}}{2} \gamma_{\mu}
\end{aligned}
$$

where $m_{g}=800 \mathrm{MeV}$ is a gluon mass scale generated dynamically in QCD [32], and $\alpha_{\mathrm{IR}}$ is the CI model parameter, which can be interpreted as the interaction strength in the infrared region [33, 34].

With this interaction, a constant mass function is obtained. Since it is needed to solve divergent integrals, a regularization procedure should be adopted. To regularize the integrals in the CI model, the proper time regularization scheme [15] is adopted. This leads to a quark propagator:

$$
S_{f}^{-1}(p)=i \gamma \cdot p+M_{f}
$$

with a constant function:

$$
M_{f}=m_{f}+\frac{M_{f}^{3}}{3 \pi^{2} m_{G}^{2}} \Gamma\left(-1, \tau_{\mathrm{UV}} M_{f}^{2}, \tau_{\mathrm{IR}} M_{f}^{2}\right),
$$

where $\Gamma\left(a, z_{1}, z_{2}\right)$ is the generalized incomplete Gamma function:

$$
\Gamma\left(a, z_{1}, z_{2}\right)=\Gamma\left(a, z_{1}\right)-\Gamma\left(a, z_{2}\right)
$$

The parameters $\tau_{\mathrm{IR}}$ and $\tau_{\mathrm{UV}}$ are infrared and ultraviolet regulators, respectively. A nonzero value for $\tau_{\mathrm{IR}} \equiv 1 / \Lambda_{\mathrm{IR}}$ implements confinement [35]. On the other hand, since the CI model represents a nonrenormalizable theory, $\tau_{\mathrm{UV}} \equiv 1 / \Lambda_{\mathrm{UV}}$ becomes part of the model and therefore sets the scale for all dimensional quantities.

\subsection{Bethe-Salpeter equation}

The bound-state problem for hadrons characterized by two valence-fermions may be studied using the homogeneous BS equation [36]:

$$
[\Gamma(k ; P)]_{t u}=\int \frac{d^{4} q}{(2 \pi)^{4}}\left[\chi_{f g}(q ; P)\right]_{s r} K_{t u}^{r s}(q, k ; P),
$$

where $\Gamma$ is the bound-state's BS amplitude; $\chi_{f g}(q ; P)=S_{f}(q+P) \Gamma S_{g}(q)$ is the BS wave-function; $r, s, t, u$ represent colour, flavor and spinor indices; $S_{f, g}$ is the quark propagator with flavor $f$ or $g$, and $K$ is the relevant fermion-fermion scattering kernel. This equation possesses solutions on that discrete set of $P^{2}$-values for which bound-states exist. A detailed description on the computation of mesons masses for pseudoscalar, vector, scalar and axial-vector mesons are explained by Bedolla et al. [16]; for diquarks and baryons are found in [22]. We will describe the spectroscopy of charming beauty mesons in Section 3. 
Table 1: Dimensionless coupling constant $\hat{\alpha}=\hat{\alpha}_{\mathrm{IR}} \Lambda_{\mathrm{U} V}^{2}$, where $=\alpha_{\mathrm{IR}} / m_{g}^{2}$, for the CI model, extracted from a best-fit to data, as explained by Raya et al. [17]. To find unequal mass systems parameters, $\Lambda_{\mathrm{IR}}$ and $\alpha$ are computed through equations (7)-(8) by fixing the pseudoscalar heavy-light meson experimental mass value. Fixed parameters are $m_{g}=800 \mathrm{MeV}$ and $\Lambda_{\mathrm{IR}}=0.24 \mathrm{GeV}$.

\begin{tabular}{lcc}
\hline \hline \multicolumn{3}{c}{ Contact Interaction Parameters } \\
\hline quarks & $\hat{\alpha}_{\mathrm{IR}}\left[\mathrm{GeV}^{-2}\right]$ & $\Lambda_{\mathrm{UV}}[\mathrm{GeV}]$ \\
$u, d, s$ & 4.565 & 0.905 \\
$c, d, s$ & 0.962 & 1.322 \\
$c$ & 0.222 & 2.305 \\
$b, u, s$ & 0.177 & 2.522 \\
$b, c$ & 0.049 & 4.131 \\
$b$ & 0.017 & 6.559 \\
\hline \hline
\end{tabular}

\subsection{CI model running coupling}

The dressed mass of quarks up/down and strange can be obtained through equation (4) and the first parameters on Table 1, and quarks charm and bottom with the respective parameters from the same table. Dressed quark masses are listed in Table 2. Once computed, it is possible to compute the meson masses through equation (6) along with their respectives Bethe-Salpeter amplitudes [16]. As reported by Chen et al. [14], when studying the spectrum of light mesons with strangeness, it is possible to found the values of the coupling $\alpha_{\mathrm{IR}}$, the ultraviolet and infrared cutoff $\Lambda_{\mathrm{UV}}$ and $\Lambda_{\mathrm{IR}}$ of the CI model to study the light sector.

In attempting to study charmonia with these same parameters, Bedolla et al. [16] found that a change in the model parameters is required: an increment in the ultraviolet regulator, and a reduction in the coupling strength. Later, Raya et al. [17] expanded that picture to examine bottomonia and concluded that a different set of parameters are needed in order to study each meson sector: light, charm and bottom.

With these set of parameters, a dimensionless coupling can be defined in terms of the ultraviolet cutoff:

$$
\alpha=\alpha\left(\Lambda_{\mathrm{UV}}\right)=\frac{\alpha_{\mathrm{IR}}}{m_{g}^{2}} \Lambda_{\mathrm{UV}}^{2}
$$

It is interesting that this dimensional coupling can be fitted by an inverse logarithmic curve, as a reminiscent of the running coupling QCD with the momentum scale at which it is measured:

$$
\alpha\left(\Lambda_{\mathrm{UV}}\right)=a \ln ^{-1}\left(\Lambda_{\mathrm{UV}} / \Lambda_{0}\right)
$$

where $a=0.923$ and $\Lambda_{0}=0.357 \mathrm{GeV}$ [17]. With this fit, the value of the strength coupling $\alpha$ can be recovered once a value of $\Lambda_{\mathrm{UV}}$ is given. The infrared cutoff $\Lambda_{\mathrm{UV}}$ and the gluon mass $m_{g}$ maintained the same values of the light sector. 
Table 2: Computed dressed-quark masses: $M_{u, s}$ are computed with light parameters, $M_{c}$ with charm parameters and $M_{b}$ with bottom parameters from Table 1.

\begin{tabular}{cccc}
\hline \hline \multicolumn{4}{c}{ Quark masses $[\mathrm{GeV}]$} \\
\hline$m_{u}=0.007$ & $m_{s}=0.170$ & $m_{c}=1.080$ & $m_{b}=3.946$ \\
$M_{u}=0.367$ & $M_{s}=0.530$ & $M_{c}=1.525$ & $M_{b}=4.675$ \\
\hline \hline
\end{tabular}

\section{Charming bound states}

The analytic expression used to compute the spectrum of mesons are found in [16]. Moreover, to study scalar and axial-vector mesons, the coupling strength provided in Table 1 is reduced by the following spin-orbit (SO) parameters:

$$
g_{\mathrm{S} O}^{1^{+}}=0.312 / 3, \quad g_{\mathrm{S} O}^{0^{+}}=0.25 / 3 .
$$

These values could be interpreted as a reduction of the coupling due to the effects of a chromomagnetic cloud which reduces the coupling in axial-vector and scalar mesons [14]. With similar values, Lu et al. [20] obtained the correct mass splitting $m_{a_{1}}-m_{\rho}=0.45 \mathrm{GeV}$ and $m_{\sigma}-m_{\rho}=0.29 \mathrm{GeV}$.

Parameters used in the contact interaction model are in Table 1, and dressed quark masses set in the quark propagator obtained via equation (4) are listed in Table 2. The spectrum of mesons are listed in Tables 3-6. In these tables, it can be seen that the mass spectrum is relatively close to experimental values. Actually, the overall value is in accordance within a $2 \%$ from experiment.

Table 3: Computed masses for pseudoscalar mesons (in $\mathrm{GeV}$ ) with the parameters in Table 1. Experimental masses are taken from [26, 27].

\begin{tabular}{cccccccc}
\hline \hline Meson & $D^{0}(c \bar{u})$ & $D_{s}^{+}(c \bar{s})$ & $\eta_{c}(c \bar{c})$ & $B^{+}(u \bar{b})$ & $B_{s}^{0}(s \bar{b})$ & $B_{c}^{+}(c \bar{b})$ & $\eta_{b}(b \bar{b})$ \\
PDG(2020) & 1.864 & 1.968 & 2.984 & 5.279 & 5.367 & 6.275 & 9.40 \\
CI Model & 1.864 & 1.954 & 2.979 & 5.279 & 5.366 & 6.275 & 9.438 \\
\hline \hline
\end{tabular}

Table 4: Computed masses for vector mesons (in $\mathrm{GeV}$ ) with the parameters in Table 1. Experimental masses are taken from [26, 27].

\begin{tabular}{cccccccc}
\hline \hline Meson & $D^{* 0}(c \bar{u})$ & $D_{s}^{*}(c \bar{s})$ & $J / \psi(c \bar{c})$ & $B^{+*}(u \bar{b})$ & $B_{s}^{0 *}(s \bar{b})$ & $B_{c}^{*}(c \bar{b})$ & $\Upsilon(b \bar{b})$ \\
PDG(2020) & 2.010 & 2.112 & 3.096 & 5.324 & 5.415 & $\ldots$ & 9.460 \\
CI model & 2.053 & 2.140 & 3.143 & 5.321 & 5.410 & 6.310 & 9.460 \\
\hline \hline
\end{tabular}


Table 5: Computed masses for scalar mesons (in GeV) with the parameters in Table 1. Experimental masses are taken from $[26,27]$.

\begin{tabular}{cccccccc}
\hline \hline Meson & $D_{0}^{* 0}(c \bar{u})$ & $D_{s 0}^{*}(c \bar{s})$ & $\chi_{c 0}(c \bar{c})$ & $B_{0}^{ \pm}(u \bar{b})$ & $B_{s 0}^{0}(s \bar{b})$ & $B_{c 0}^{+}(c \bar{b})$ & $\chi_{b 0}(b \bar{b})$ \\
PDG(2020) & 2.300 & 2.318 & 3.415 & $\ldots$ & $\ldots$ & $\ldots$ & 9.859 \\
CI model & 2.458 & 2.555 & 3.442 & 5.589 & 5.679 & 6.492 & 9.586 \\
\hline \hline
\end{tabular}

Table 6: Computed masses for axial-vector mesons (in $\mathrm{GeV}$ ) with the parameters in Table 1. Experimental masses are taken from [26, 27].

\begin{tabular}{cccccccc}
\hline \hline Meson & $D_{1}^{0}(c \bar{u})$ & $D_{s 1}^{ \pm}(c \bar{s})$ & $\chi_{c 1}(c \bar{c})$ & $B_{1}^{+, 0}(u \bar{b})$ & $B_{s 1}^{0}(s \bar{b})$ & $B_{c 1}^{+}(c \bar{b})$ & $\chi_{b 1}(b \bar{b})$ \\
PDG(2020) & 2.421 & 2.459 & 3.511 & 5.726 & 5.829 & $\ldots$ & 9.893 \\
CI model & 2.529 & 2.620 & 3.465 & 5.639 & 5.724 & 6.519 & 9.595 \\
\hline \hline
\end{tabular}

\section{Conclusions and Final Remarks}

The general details about the CI model were presented, along with a brief description on how to implement it to compute the spectrum of mesons. It was found that the results obtained are in agreement within a $2 \%$ when compared with experimental data. Due to the precision on the prediction of vector meson masses, it is expected that a future measurement of the mass of $B_{c}^{*}(c \bar{b})$ will very close to our predicted value. Future works include studying electromagnetic form factors of heavy-light systems [37] and the computation of spectrum of fully-heavy tetraquarks [38].

\section{References}

[1] P. Jain and H. J. Munczek, Phys. Rev. D 48, 5403-5411 (1993) doi:10.1103/PhysRevD.48.5403 [arXiv:hep-ph/9307221 [hep-ph]].

[2] C. D. Roberts and A. G. Williams, Prog. Part. Nucl. Phys. 33, 477-575 (1994) doi:10.1016/0146-6410(94)90049-3 [arXiv:hep-ph/9403224 [hep-ph]].

[3] A. Bashir, L. Chang, I. C. Cloet, B. El-Bennich, Y. X. Liu, C. D. Roberts and P. C. Tandy, Commun. Theor. Phys. 58, 79-134 (2012) doi:10.1088/0253-6102/58/1/16 [arXiv:1201.3366 [nucl-th]].

[4] P. Maris and P. C. Tandy, Phys. Rev. C 62, 055204 (2000) doi:10.1103/PhysRevC.62.055204 [arXiv:nucl-th/0005015 [nucl-th]].

[5] J. Chen, M. Ding, L. Chang and Y. x. Liu, Phys. Rev. D 95, no.1, 016010 (2017) doi:10.1103/PhysRevD.95.016010 [arXiv:1611.05960 [nucl-th]].

[6] N. Nakanishi, Phys. Rev. 130, 1230-1235 (1963) doi:10.1103/PhysRev.130.1230 
[7] L. Chang, I. C. Cloët, C. D. Roberts, S. M. Schmidt and P. C. Tandy, Phys. Rev. Lett. 111, no.14, 141802 (2013) doi:10.1103/PhysRevLett.111.141802 [arXiv:1307.0026 [nucl-th]].

[8] K. Raya, L. Chang, A. Bashir, J. J. Cobos-Martinez, L. X. Gutiérrez-Guerrero, C. D. Roberts and P. C. Tandy, Phys. Rev. D 93, no.7, 074017 (2016) doi:10.1103/PhysRevD.93.074017 [arXiv:1510.02799 [nucl-th]].

[9] K. Raya, M. Ding, A. Bashir, L. Chang and C. D. Roberts, Phys. Rev. D 95, no.7, 074014 (2017) doi:10.1103/PhysRevD.95.074014 [arXiv:1610.06575 [nucl-th]].

[10] L. X. Gutierrez-Guerrero, A. Bashir, I. C. Cloet and C. D. Roberts, Phys. Rev. C 81, 065202 (2010) doi:10.1103/PhysRevC.81.065202 [arXiv:1002.1968 [nucl-th]].

[11] H. L. L. Roberts, C. D. Roberts, A. Bashir, L. X. Gutierrez-Guerrero and P. C. Tandy, Phys. Rev. C 82, 065202 (2010) doi:10.1103/PhysRevC.82.065202 [arXiv:1009.0067 [nucl-th]].

[12] H. L. L. Roberts, L. Chang, I. C. Cloet and C. D. Roberts, Few Body Syst. 51, 1-25 (2011) doi:10.1007/s00601-011-0225-x [arXiv:1101.4244 [nucl-th]].

[13] H. L. L. Roberts, A. Bashir, L. X. Gutierrez-Guerrero, C. D. Roberts and D. J. Wilson, Phys. Rev. C 83, 065206 (2011) doi:10.1103/PhysRevC.83.065206 [arXiv:1102.4376 [nucl-th]].

[14] C. Chen, L. Chang, C. D. Roberts, S. Wan and D. J. Wilson, Few Body Syst. 53, 293-326 (2012) doi:10.1007/s00601-012-0466-3 [arXiv:1204.2553 [nucl-th]].

[15] D. Ebert, T. Feldmann and H. Reinhardt, Phys. Lett. B 388, 154-160 (1996) doi:10.1016/03702693(96)01158-6 [arXiv:hep-ph/9608223 [hep-ph]].

[16] M. A. Bedolla, J. J. Cobos-Martínez and A. Bashir, Phys. Rev. D 92, no.5, 054031 (2015) doi:10.1103/PhysRevD.92.054031 [arXiv:1601.05639 [hep-ph]].

[17] K. Raya, M. A. Bedolla, J. J. Cobos-Martínez and A. Bashir, Few Body Syst. 59, no.6, 133 (2018) doi:10.1007/s00601-018-1455-y [arXiv:1711.00383 [nucl-th]].

[18] M. A. Bedolla, E. Santopinto and L. X. Gutiérrez-Guerrero, EPJ Web Conf. 192, 00039 (2018) doi:10.1051/epjconf/201819200039 [arXiv:1810.04391 [hep-ph]].

[19] M. A. Bedolla and E. Santopinto, Springer Proc. Phys. 238, 737-743 (2020) doi:10.1007/9783-030-32357-8_118

[20] Y. Lu, C. Chen, C. D. Roberts, J. Segovia, S. S. Xu and H. S. Zong, Phys. Rev. C 96, no.1, 015208 (2017) doi:10.1103/PhysRevC.96.015208 [arXiv:1705.03988 [nucl-th]].

[21] P. L. Yin, C. Chen, G. Krein, C. D. Roberts, J. Segovia and S. S. Xu, Phys. Rev. D 100, no.3, 034008 (2019) doi:10.1103/PhysRevD.100.034008 [arXiv:1903.00160 [nucl-th]].

[22] L. X. Gutiérrez-Guerrero, A. Bashir, M. A. Bedolla and E. Santopinto, Phys. Rev. D 100, no.11, 114032 (2019) doi:10.1103/PhysRevD.100.114032 [arXiv:1911.09213 [nucl-th]]. 
[23] M. A. Bedolla, K. Raya, J. J. Cobos-Martínez and A. Bashir, Phys. Rev. D 93, no.9, 094025 (2016) doi:10.1103/PhysRevD.93.094025 [arXiv:1606.03760 [hep-ph]].

[24] M. A. Bedolla, Few Body Syst. 60, no.2, 24 (2019) doi:10.1007/s00601-019-1487-y

[25] G. Eichmann, C. S. Fischer and W. Heupel, Phys. Lett. B 753, 282-287 (2016) doi:10.1016/j.physletb.2015.12.036 [arXiv:1508.07178 [hep-ph]].

[26] C. Patrignani et al. [Particle Data Group], Chin. Phys. C 40, no.10, 100001 (2016) doi:10.1088/1674-1137/40/10/100001

[27] M. Tanabashi et al. [Particle Data Group], Phys. Rev. D 98, no.3, 030001 (2018) doi:10.1103/PhysRevD.98.030001

[28] T. Nguyen, N. A. Souchlas and P. C. Tandy, AIP Conf. Proc. 1361, no.1, 142-151 (2011) doi:10.1063/1.3622693 [arXiv:1005.3315 [nucl-th]].

[29] C. S. Fischer, S. Kubrak and R. Williams, Eur. Phys. J. A 51, 10 (2015) doi:10.1140/epja/i201515010-7 [arXiv:1409.5076 [hep-ph]].

[30] M. Gómez-Rocha, T. Hilger and A. Krassnigg, Phys. Rev. D 93, no.7, 074010 (2016) doi:10.1103/PhysRevD.93.074010 [arXiv:1602.05002 [hep-ph]].

[31] T. Hilger, M. Gómez-Rocha, A. Krassnigg and W. Lucha, Eur. Phys. J. A 53, no.10, 213 (2017) doi:10.1140/epja/i2017-12384-4 [arXiv:1702.06262 [hep-ph]].

[32] P. Boucaud, J. P. Leroy, A. L. Yaouanc, J. Micheli, O. Pene and J. Rodriguez-Quintero, Few Body Syst. 53, 387-436 (2012) doi:10.1007/s00601-011-0301-2 [arXiv:1109.1936 [hep-ph]].

[33] D. Binosi, C. Mezrag, J. Papavassiliou, C. D. Roberts and J. Rodriguez-Quintero, Phys. Rev. D 96, no.5, 054026 (2017) doi:10.1103/PhysRevD.96.054026 [arXiv:1612.04835 [nucl-th]].

[34] A. Deur, S. J. Brodsky and G. F. de Teramond, Nucl. Phys. 90, 1 (2016) doi:10.1016/j.ppnp.2016.04.003 [arXiv:1604.08082 [hep-ph]].

[35] C. D. Roberts, Prog. Part. Nucl. Phys. 61, 50-65 (2008) doi:10.1016/j.ppnp.2007.12.034 [arXiv:0712.0633 [nucl-th]].

[36] E. E. Salpeter and H. A. Bethe, Phys. Rev. 84, 1232-1242 (1951) doi:10.1103/PhysRev.84.1232

[37] A. Bashir, M.A. Bedolla, L.X. Gutierrez-Guerrero, R. Hernandez., In Preparation.

[38] Bedolla, M.A., Santopinto E., J. Ferreti, C.D. Roberts, In Preparation. 